\title{
ONE-POINT COMPACTIFICATION ON CONVERGENCE SPACES
}

\author{
SHING S. SO
}

\author{
Central Missouri State University \\ Warrensburg, Missouri
}

(Received December 22, 1992 and in revised form February 11, 1993)

ABSTRACT. A convergence space is a set together with a notion of convergence of nets. It is well known how the one-point compactification can be constructed on noncompact, locally compact topological spaces. In this paper, we discuss the construction of the one-point compactification on noncompact convergence spaces and some of the properties of the one-point compactification of convergence spaces are also discussed.

AMS(MOS) SUBJ. CLASSFICATION: Primary: 54A20, 54B20, 54D35.

KEYWORDS: $\in$-subnet, reversible $\in$-subnet, pseudotopological, pretopological, limit superior, limit inferior, power set, compact, compactification.

\section{Preliminaries.}

The following definitions were introduced in [1] in establishing the definition of convergence spaces.

A $\underline{\text { net }}$ is a map $s$ whose domain is a directed set. Let $s$ be a net with domain $D$. If $m \in D$, then $s(m)$ will be denoted by $s_{m}$. If $D$ is directed by the relation $\geq$ and $m \in D$, then $\{n \in D \mid n \geq m\}$ will be denoted by $m D$. If $E \subseteq D$, then $E$ is cofinal in $D$ if for each $m$ in $E$, $m D \cap E \neq \emptyset$ and $E$ is residual in $D$ if for each $m$ in $E, m E=m D$. The domain and range of a function $f$ will be denoted by $D(f)$ and $R(f)$ respectively. If $X$ is a set and $s$ is a net with domain $D$, then $s$ is $\underline{\text { in }} X$ if $R(s) \subseteq X$. Suppose $s$ is a net with domain $D$ and $t$ is a net with domain $E$. Then $t$ is a subnet of $s$, denoted by $t \leq s$, if for each $n \in D$, there exists $m \in E$ such that $t(m E) \subseteq s(n D)$. A universal net is a net which has no proper subnet.

A convergence structure on the set $X$ is a class $C$ of ordered pairs such that $(1)$ if $(s, x) \in$ $C$, then $s$ is a net in $X$ and $x \in X$, and (2) if $(s, x) \in C$ and $t$ is a subnet of $s$, then $(t, x) \in C$. If $C$ is a convergence structure on $X$, then $(X, C)$ or just $X$ is called a convergence space, and the statement $s$ converges to $x$, denoted by $s \rightarrow x$, means $(s, x) \in C$. Furthermore, $X$ is compact if every net in $X$ has a convergent subnet, and $X$ is $\underline{\text { Hausdroff }}$ if no net in $X$ converges to two distinct points of $X$.

Unless it is specified, $X$ will be used to denote a convergence space throughout this paper.

The following theorem is straightforward.

THEOREM 1.1. A convergence space $X$ is compact if and only if every universal net in $X$ converges. 
Next, we introduce $\epsilon$-subnets and reversible $\epsilon$-subnets which are important in our discussion.

Let $S$ be a net of sets with domain $D$ and let $t$ be a net with domain $E$. The $t$ is called an $\underline{\epsilon-\text { subnet }}$ of $S$, denoted by $t \leq_{\epsilon} S$, if for cach $n \in D$, there exists $m \in E$ so that for each $p \geq m$ there is $q \geq n$ such that $t_{p} \in S_{q}$. Similarly, the notation $S \leq_{\ni} t$ will mean that for each $m \in E$, there exists $n \in D$ so that for cach $q \geq n$, there is $p \geq m$ such that $t_{q} \in S_{p}$. Furthermore, $t$ is called a reversible $\in-n e t$ of $S$ if $t \leq_{\epsilon} S$ and $S \leq_{\ni} t$.

Let $X$ be a convergence space, and let $S$ bc a net of sets in $X$. Then the set of all $x$ such that some $\epsilon$-subnet of $S$ converges to $x$ is called the limit superior of $S$, denoted by $\lim \sup S$, and the set of all $x$ such that some reversible $\in$-subnet of $S$ converges to $x$ is called the limit inferior of $S$, denoted by $\lim \inf S$.

Z. Frolik [2] established the following theorem for topological spaces and So [3] showed that the result also holds for convergence spaces.

THEOREM 1.2. If $X$ is a convergence space, $S$ is a net of sets such that for each $n \in D(S)$ $S_{n} \subseteq X$, and $T \leq S$, then $\lim$ inf $S \subseteq \lim$ inf $T \subseteq \lim$ sup $T \subseteq \lim$ sup $S$.

The next lemma follows immediately from the definitions involved.

LEMMA 1.1. Suppose $s$ is a net and $T$ and $S$ are net of sets. If $s \leq_{\epsilon} T$ and $T \in S$, then $s \leq_{\in} S$.

LEMMA 1.2. If $s \leq_{\epsilon} S$, then there is a subnet $U$ of $S$ such that $s$ is a reversible $\epsilon$-subnet of $U$.

PROOF. Let $D=D(S)$ and $E=D(s)$. Let $F=\left\{(m, n) \in D \times E \mid s_{n} \in S_{m}\right\}$ with the cross product order. Then $F$ is a directed set.

Let $U$ be the net with domain $F$ defined by $U(m, n)=S_{m}$. Let $p \in D$. Since $s \leq \epsilon S$, there exists $q \in E$ such that every element of $s(q E)$ is belongs to some element of $S(p D)$. Let $(m, n) \geq(p, q)$. Then we have $m \geq p$ and $U(m, n)=S_{m}$. Thus $U \leq S$.

Let $k \in E$. Since $s \leq_{\epsilon} S$, there exists $q \geq k$ such that $s_{q} \in U(p, q)$ for some $p$ in $D$. Let $(m, n) \geq(p, q)$. Then we have $n \geq k$ and $s_{n} \in U(m, n)$. Therefore $U \leq_{\ni} s$.

Let $(h, k) \in F$. Since $s \leq_{\epsilon} S$, there cxists $p \in E$ such that if $n \geq p$, there is $m \geq h$ such that $s_{n} \in U(m, n)$. Let $q \in E$ such that $q \geq k$ and $q \geq p$. Hence $q \in k E$ such that if $j \geq q$, there is $i \geq h$ such that $s, \in U(i, j)$, i.e., if $j \geq q$, there is $(i, j) \geq(h, q) \geq(h, k)$ such that $s, \in U(i, j)$. Therefore $s \leq_{\epsilon} U$.

THEOREM 1.3. If $S$ is universal, then $\lim \sup S=\lim$ inf $S$.

PROOF. Let $x \in \lim \sup S$. Then there exists an $\in$-subnet $s$ of $S$ such that $s \rightarrow x$. By Lemma 1.2, $S \leq_{\ni} s$. Therefore $s$ is a reversible $\epsilon$-subnet of $S$ and hence $x \in \lim$ inf $S$. Since $\lim$ sup $S \subseteq \lim$ inf $S$, it follows from Theorem 1.2 that lim sup $S=\lim$ inf $S$.

If $X$ is a set and $s$ is a net with domain $D$, then $s$ is in $X$ if $R(s) \subseteq X, s$ is eventually in $X$ if for some $m$ in $D, s(m D) \subseteq X$, and $s$ is frequently in $X$ if for each $m \in D s(m D) \cap X \neq \emptyset$

LEMMA 1.3. If $s$ is a net in $X$ and $A$ is a nonempty subset of $X$, then $s$ is frequently in $A$ if and only if some subnet of $s$ is eventually in $A$. 
PROOF. Suppose $s$ is frequently in $A$. Let $D=D(s)$. Then for each $i$ in $D, s(i D) \cap A \neq \emptyset$. Let $m \in D$ and $E=\left\{n \in m D \mid s_{n} \in s(m D) \cap A\right\}$. Then $E$ is a dirccted set. Let $u=s \mid E$, and let $\imath \in m D$. Then there exists $\jmath \in E$ such that $u(j E) \subseteq s(i D) \cap A$ and hence $u(j E) \subseteq s(i D)$ and $u(j E) \subseteq A$. Therefore $u \leq s$ and $u$ is eventually in $A$.

Suppose some subnct $u$ of $s$ is cventually in $A$. Let $D=D(s), E=D(u)$, and $n \in D$. Then there exists $m \in E$ such that $u(m E) \subseteq s(n E)$. Since $u$ is eventually in $A$, there exists $i \in E$ such that $u(i E) \subseteq A$. Let $\jmath \geq m$ and $j \geq \imath$. Then $u(j E) \subseteq s(n E) \cap A$. Therefore $s$ is frequently in $A$.

The following lemma is Proposition 3.3 of [4].

LEMMA 1.4. If $s$ is a net in the set $X$, then $s$ is universal if and only if for each subset $Y$ of $X, s$ is eventually in $Y$ or cventually in $X-Y$.

\section{THE POWER SET AND THE ONE-POINT COMPACTIFICATION OF $X$}

Let $\mathcal{P} X$ denote the power set of $X$. If $X$ is a convergence space, $L \in \mathcal{P} X$, and $S$ is a net in $\mathcal{P} X$, then the statement that $S \rightarrow L$ in $\mathcal{P} X$ means that $L=\lim \sup S=\lim$ inf $S$.

It follows from Theorem 1.2 that $\mathcal{P} X$ is a convergence spacc.

In this section, we investigate the convergence structure of the power set, $\mathcal{P} X$, of a convergence space $X$ and the construction of the one-point compactification $X^{*}$ of $X$. We then show that $X^{*}$ is homeomorphic to a subspace of $\mathcal{P} X^{*}$.

It should be noted that in [5] and [6], G.D. Richardson and D.C. Kent studied the one-point compactification and the star compactification on convergence spaces defined by filters. There are some similarities between the construction of the one-point compactification of convergence spaces defined by filters and convergence spaces defined. One of the essential differences is that the convergence structure defined by filters has the constant convergence property built in the definition, while in the convergence structure defincd by nets the constant convergence property is not assumed.

Let $f$ be a map from $X$ to $Y$. The statement $f$ is continuous means that if $s \rightarrow x$ in $X$, then $f \circ s \rightarrow f(x)$. The statcment that $f$ is a homeomorphism means that $f$ is one-to-one, onto, continuous, and $f^{-1}$ is continuous. $X$ is said to be homeomorphic to $Y$ if there is a homcomorphism from $X$ onto $Y$.

THEOREM 2.1. If $X$ is Hausdorff and $X^{\prime}=\{\{x\} \mid x \in X\}$, then $X$ is homeomorphic to the subspace $X^{\prime}$ of $\mathcal{P} X$.

PROOF. Let $h$ be a map from $X$ to $X^{\prime}$ such that for cach $x \in X, h(x)=\{x\}$. Then it is obvious that $h$ is onc-to-onc and onto. Let $s \rightarrow x$. Then $s$ is a reversible $\in$-subnet of $h \circ s$ and hence $x \in \lim$ inf $h \circ s$. Since $h(x)=\{x\}, h(x) \subseteq \lim$ inf $h \circ s$. Let $y \in \lim$ sup $h \circ s$. Then there exists an $\epsilon$-subnet $t$ of $h \circ s$ such that $t \rightarrow y$. Since $X$ is Hausdorff and $t \leq s, x=y$ and hence $y \in h(x)$. Thereforc $\lim$ sup $h \circ s \subseteq h(x) \subseteq \lim$ inf $h \circ s$. It follows from Theorem 1.2 that $h \circ s \rightarrow h(s)$. Let $S \rightarrow\{x\}$ in $X^{\prime}$. Then there exists a reversible $\epsilon$-subnet $u$ of $S$ with domain $E$ such that $u \rightarrow x$. Let $v=h^{-1} \circ S$ and $D=D(S)$. Let $n \in E$. Then there exists $m \in D$ such that if $p \geq m$, there is $q \geq n$ such that $S_{p} \ni u_{q}$ and hence $v_{p}=h^{-1}\left(S_{p}\right)=u_{q}$. 
Therefore $v \leq u$ and hence $h^{-1} \circ S \rightarrow h^{-1}(\{x\})$. Since $h$ is onc-to-onc and onto, and both $h$ and $h^{-1}$ are continuous, $X^{\prime}$ is homemorphic to the subspace $X^{\prime}$ of $\mathcal{P} X$.

Let $X$ be a convergence spiace. If $M \subseteq X$, then $\bar{M}$ is the set of all points $x$ such that some net in $M$ converges to $x$. A sct $D$ is drnsor $m . X$ if $\bar{D}=X$. A compactifacation of a convergence space $X$ is an ordered pair $(Y, h)$ such that $Y$ is compact, $h$ is a homeomorphism of $X$ into $Y$, and $h(X)$ is dense in $Y$.

Let $X$ be a noncompact convergence space, and let $\infty$ be a point not in $X$. Let $X^{*}=$ $X \cup\{\infty\}$. Vicwing the result in Lemma 1.3, we clefine the convergence in $X^{*}$ as follows. Suppose $s$ is a net in $X^{*}$. Let $s \rightarrow x$ in $X^{*}$ for some $x$ in $X$ if and only if $s$ is frequently in $X$ and $s \mid X \rightarrow x$, and let $s \rightarrow \infty$ in $X^{*}$ if and only if no subnet of $s$ in $X$ converges in $X$.

Suppose $s$ is a universal net in $X$. Then by Lemma 1.4, s is eventually in $X$ or $\{\infty\}$. If $s$ is evcntually in $\{\infty\}$ or $s \mid X \nrightarrow x$ for any $x$ in $X$, then no subnet of $s$ in $X$ converges in $X$ and hence $s \rightarrow \infty$. Otherwise $s \rightarrow x$ for some $x$ in $X$ according to the convergence defined on $X^{*}$. Consequently, we have the following theorem.

THEOREM 2.2. If $X$ is a noncompart convergence space, then $X^{*}$ is a compactification of $X$.

THEOREM 2.3. If $X^{*}$ is a noncompact, Hausdorff convergence space, then $X^{*}$ is homeomorphic to the subspace $\{\{x\} \mid x \in X\} \cup\{\emptyset\}$ in $\mathcal{P}(X \cup\{\infty\})$.

PROOF. Let $X^{\prime}=\{\{x\} \mid x \in X\}$, and let $h^{\prime}$ be the map from $X^{*}$ to $X^{\prime} \cup\{\emptyset\}$ such that for each $x$ in $X, h^{\prime}(x)=\{x\}$, and $h^{\prime}(\infty)=\emptyset$. Notc that $h^{\prime}$ is an extcnsion of the homeomorphism $h$ in Theorem 2.1. It is clear that $h^{\prime}$ is one-to-one and onto. Suppose $s \rightarrow \infty$. Let $u$ be an $\epsilon$-subnct of $h^{\prime} \circ s$. Then $u \mid X \nrightarrow x$ for each $x$ in $X$. Therefore lim sup $h^{\prime} \circ s=\emptyset$. Thus $h^{\prime}$ is strongly continuous. Let $S$ be a net in $X^{\prime} \cup\{\emptyset\}$ with domain $D$ and $s=h^{\prime-1} \circ S$. Suppose $S \rightarrow\{x\}$ for some $x$ in $X$. Then there cxists a reversible $\epsilon$-subnet $u$ of $S$ with domain $E$ such that $u \rightarrow x$. Let $n \in E$. Then there exists $m \in D$ such that if $p \geq m$, there is $q \geq n$ such that $S_{p} \ni u_{q}$ and hence $s_{p}=u_{q}$. Therefore $s \leq u$ and thus $s \rightarrow x$. Hence $h^{\prime-1} \circ S \rightarrow h^{\prime-1}(\{x\})$. Suppose $S \rightarrow \emptyset$. Then lim sup $S=\lim$ inf $S=\emptyset$ and hence no $\epsilon$-subnet of $S$ converges in $X$. Now $s=h^{\prime-1} \circ S$. Let $v \leq s$. Then $v \leq_{\epsilon} S$ and hence $v$ does not converges in $X$. Therefore $s \rightarrow \infty$ and thus $h^{\prime-1} \circ S \rightarrow h^{\prime-1}(\emptyset)$. Therefore $h^{\prime}$ is a homeomorphism, and hence $X^{*}$ is homeomorphic to $X^{\prime} \cup\{\emptyset\}$.

A net of nets with domain $D$ is a net $s$ with domain $D$ such that for each $n \in D, s_{n}$ is a net. If $s$ is a net of nets with domain $D$ and for each $n \in D, D\left(s_{n}\right)=D_{n}$, then the diagonal net generated by $s$, denoted by $\Delta s$, is the net $t$ such that $D(t)=D \times \Pi\left\{D_{n} \mid n \in D\right\}$ with the cross product order and for each $(n, f) \in D(t), t(n, f)=s_{n}(f(n))$.

The statement that $X$ is pseudotopological at $x$ mcans that if $s$ is a net in $X$ such that each universal subnet of $s$ converges to $x$, then $s \rightarrow x$. The statement that $X$ is pretopological at $x$ means that if $s$ is a net of nets in $X$ with domain $D$ such that for each $n \in D, s_{n} \rightarrow x$, then $\Delta s \rightarrow x$.

THEOREM 2.4. If $X$ is Hausdorff and pseudotopological, then $X^{*}$ is pseudotopological. 
PROOF. Let $x \in X^{*}$ and let $s$ be a net in $X^{*}$ such that every universal subnet of $s$ converges to $x$ in $X^{*}$. Suppose $x$ is in $X$. Let $u$ be a universal subnet of $s$. Since $u \rightarrow x, u$ is frequently in $X$ and $u \mid X \rightarrow x$. By Lemma 1.3, $u$ is eventually in $X$. According to Theorem $1.4, s$ is frequently in $X$. Let $v$ be a universal subnet of $s \mid X$. Then $v$ is a universal subnet of $s$ and hence $v \rightarrow x$. Since $X$ is pseudotopological, $s \mid X \rightarrow x$ and hence $s \rightarrow x$. Suppose $x=\infty$. Let $t$ be a subnet of $s$. Suppose $t$ converges to some point $y$ in $X$. Let $u$ be a universal subnet of $t$. Since $u \rightarrow x, X$ is Hausdorff, $u \leq t$, and $t \rightarrow y$, it follows that $y=x$. This contradicts the supposition that $x=\infty$. Therefore no subnet of $s$ converges in $X$ and hence $s \rightarrow \infty$. Thus $X^{*}$ is pseudotopological.

The following three lemmas are Theorems 14,4 , and 8 in [1].

LEMMA 2.1. In order that the convergence space $X$ is pretopological at $x$ it is necessary and sufficient that if $s$ is a universal net of universal nets in $X$ such that for each $n$ in $D(s)$, $s_{n} \rightarrow x$, then $\Delta s \rightarrow x$.

LEMMA 2.2. If $s$ is a net of nets with domain $D$ and $t \leq \Delta s$, then there exist a cofinal subset $E$ of $D$ and a net $u$ of nets with domain $E$ such that for each $n$ in $E, u_{n} \leq s_{n}$ and $\Delta u \leq t$

LEMMA 2.3. If $s$ is a net with domain $D$ and $t$ is $\mathrm{n}$ net of nets with domain $D$ such that for each $n \in D t_{n}$ is eventually in $s(n D)$, then $\Delta t \leq s$.

The following example shows that although a convergence space is pretopological, its onepoint compactification may not be pretopologcial.

EXAMPLE. Let $X=\{1,2,3, \cdots\}$ and let $s \rightarrow x$ if and only if $s$ is eventually in $\{x\}$ for each net $s$ in $X$ and $x \in X$. Suppose $u$ is a net of nets in $X$ with domain $D$ such that $u_{n} \rightarrow x$ for each $n \in D$. Let $v$ be a net with domain $D$ such that $v_{n}=x$ for each $n \in D$. By Lemma 2.3, $\Delta u \leq v_{n}$ for each $n \in D$. Therefore $\Delta u \rightarrow x$ and hence $X$ is pretopological.

Let $X \cup\{\infty\}$ be the one-point compactification of $X$, and let $s$ be the net nets with domain $N$, the set of natural number, such that for each $n \in N$, the domain of the net $s_{n}$ is $N$ also.

If $n$ is odd, define $s_{n}(m)= \begin{cases}1 & \text { if } m \leq n \\ (m+1)-n & \text { if } n>m .\end{cases}$

If $n$ is even, define $s_{n}(m)=\infty$ for every $n \in N$. Let $t$ be a net with domain $N$ and let

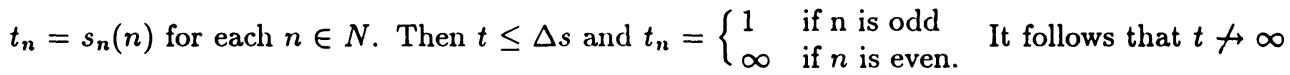
since $t \mid X$ is a subnet of $t$ such that $t \mid X \rightarrow 1$. Therefore $\Delta s \nrightarrow \infty$ and hence the one-point compactification of $X$ is not pretopological.

THEOREM 2.5. Let $X$ be a Hausdorff, pretopological convergence space, and $X^{*}$ be the one-point compactification of $X$. Suppose for every net of nets $s$ in $X^{*}$ with domain $D, s$ satisfies the condition that if for each $n \in D, s_{n} \rightarrow \infty, \Delta x \rightarrow \infty$. Then $X^{*}$ is pretopological.

PROOF. Let $s$ be a universal net of universal nets in $X^{*}$ with domain $D$ such that for each $n$ in $D s_{n} \rightarrow x$ in $X^{*}$. Suppose $x \in X$. Then for each $n \in D$ some subnet $t_{n}$ of $s_{n}$ is eventually in $X$ and hence $s_{n} \mid X \rightarrow x$. Let $u$ be the net with domain $D$ such that for each $n \in D, u_{n}=t_{n} \mid X$. Since for each $n \in D, u_{n} \leq s_{n}$ and so $u_{n} \rightarrow x$. Hence $\Delta s \rightarrow x$ because $X$ 
is pretopological. Since $X^{*}$ is compact and $\Delta s$ is universal, $\Delta s \rightarrow y$ for some $y$ in $X^{*}$. From Lemma 2.5 and the fact that $X^{*}$ is Hausdroff, $\Delta s \rightarrow x$.

Suppose $x=\infty$. By the assumption $\Delta s \rightarrow \infty$.

It follows from Lcmma 2.1 that $X^{*}$ is pretopological.

The following theorem is stated in [7], a proof is given for the sake of completeness.

THEOREM 2.6. If $X$ is compact, pscudotopological, and Hausdorff, then $X$ is topological if and only if $X$ is regular.

PROOF. Suppose $X$ is topological. Let $s$ be a net of nets in $X$ with domain $D$ such that $\Delta s \rightarrow x$ and for each $n$ in $D, s_{n} \rightarrow p_{n}$. Let $q$ be a universal subnet of $p$ win domain $E$. Then $q \rightarrow y$ for some $y \in X$ since $X$ is compact. Fix $m_{0} \in D$. Since $q \leq p$, there exists $n_{0} \in E$ such that for each $i \geq n_{0}$, there is $\jmath \geq m_{0}$ such that $q_{t}=p_{\jmath}$. Let $t$ be a net with domain $n_{0} E$ such that for each $i \in m_{0} E, t_{\imath}=s$, with $\jmath \in m_{0} E$. Then $t_{\imath} \rightarrow q_{\imath}$ for cach $i \in m_{0} E$. Thus $\Delta t \rightarrow y$ because $X$ is topological. Since $X$ is Hausclorff, $x=y$ and hence $q \rightarrow x$. Since every universal subnet of $p$ converges to $x$ and $X$ is pscudotopological, $p \rightarrow x$ and hence $X$ is regular.

Suppose $X$ is regular. Let $p$ be a net with domain $D$ such that $p \rightarrow x$ and let $s$ be a net of ncts with domain $D$ such that for each $n \in D, s_{n} \rightarrow p_{n}$. Let $u$ be a universal subnet of $\Delta s$. Then $u \rightarrow y$ for some $y \in X$. By Lemma 2.1, there exist a cofinal subset $F$ of $D$ and a net $V$ of nets with domain $F$ such that for each $n$ in $F, v_{n} \leq s_{n}$ and $\Delta v \leq u$. Therefore $\Delta v \rightarrow y$. Let $q=p \mid F$. Then $v_{m} \operatorname{toq}_{m}$ for every $m \in F$. Since $X$ is regular, $q \rightarrow y$. Since $X$ is Hausdorff and $q \leq p, q \rightarrow x$ and hence $u \rightarrow x$. Since $X$ is pseudotopological and every universla subset of $\Delta s$ converges to $x, \Delta s \rightarrow x$ and hence $X$ is topological.

The following corollary follows immediately form Theorems 2.6 and 2.8 .

COROLLARY. Let $X$ be a noncompact, Hausdorff, pseudotopological space, and let $X^{*}$ be its one-poit compactification. Then $X^{*}$ is topological if and only if $X^{*}$ is regular.

\section{REFERENCES}

1. PEARSON, B. J. Spaces defined by convergence classes of nets, Glasnik Matematicki, Vol.23(43) (1988), 135-142.

2. FROLIK, Z. Concerning topological convcrgence of sets, Czechoslovak Math. Vol.10(85) (1960), 168-180.

3. SO, S. S. Special mappings defined on convergence spaces, Journal of the Institute of Mathematics and Computer Science, Vol.5(1) (1992), 93-103.

4. AARNES, J. F. and ANDENAES, P. R. On nets and filters, Math. Scand. $\underline{31}$ (1972), 285-292.

5. KENT, D. C. and RICHARDSON G.D. Compactifications on convergence spaces, International Journal of Mathematics and Mathematical Sciences Vol.2(3) (1979), 345-368.

6. RICHARDSON G.D. and KENT D.C. The Star Compactifications, International Journal of Mathematics and Mathematical Sciences Vol.4(3) (1981), 451-472.

7. PEARSON, B. J. Unpublished Lerture Notes, University of Missouri-Kansas City. 


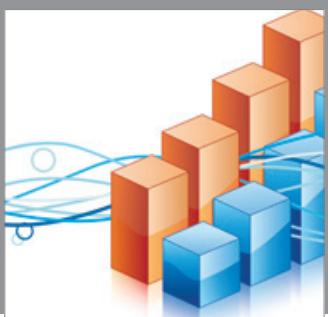

Advances in

Operations Research

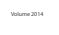

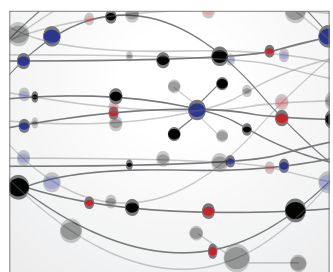

\section{The Scientific} World Journal
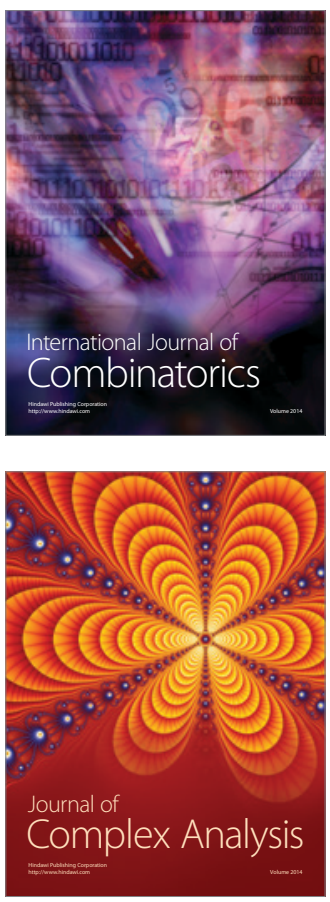

International Journal of

Mathematics and

Mathematical

Sciences
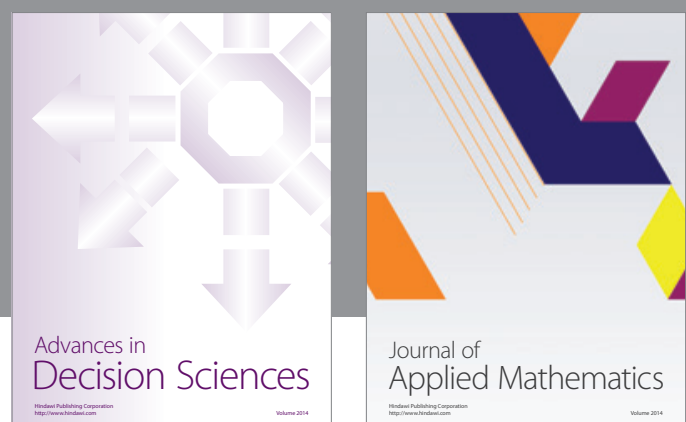

Journal of

Applied Mathematics
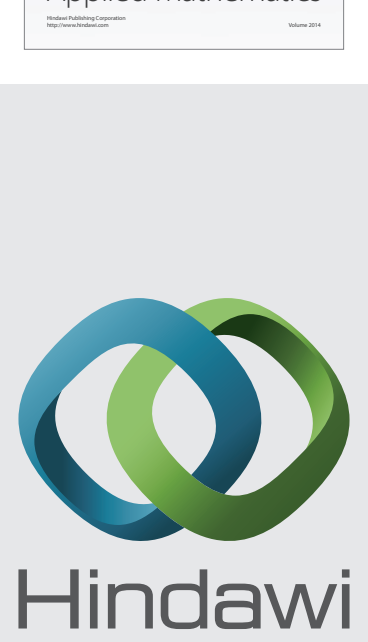

Submit your manuscripts at http://www.hindawi.com
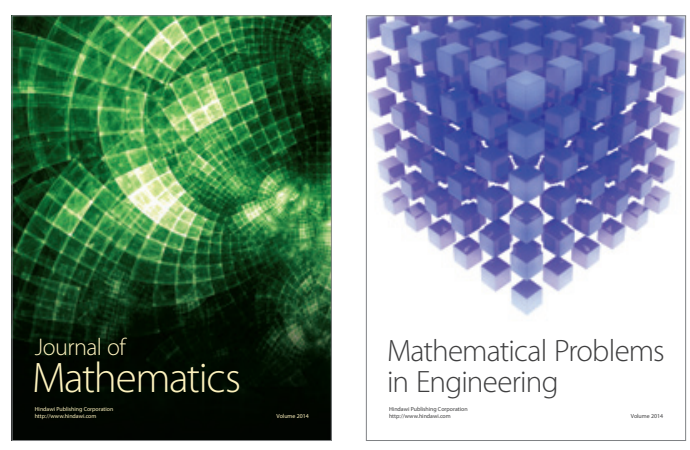

Mathematical Problems in Engineering
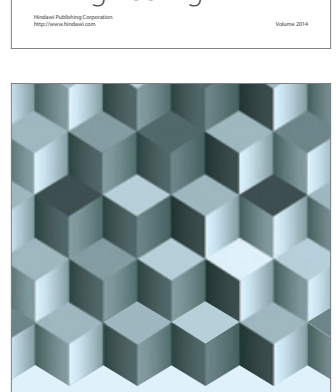

Journal of

Function Spaces
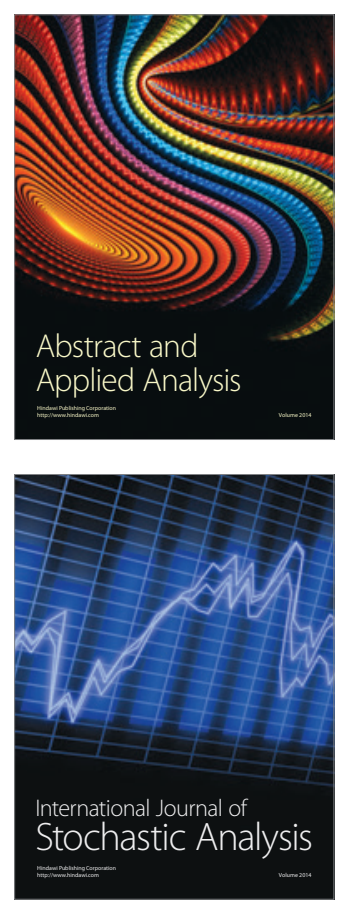

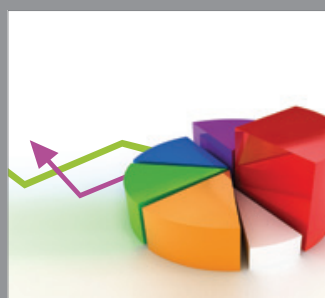

ournal of

Probability and Statistics

Promensencen
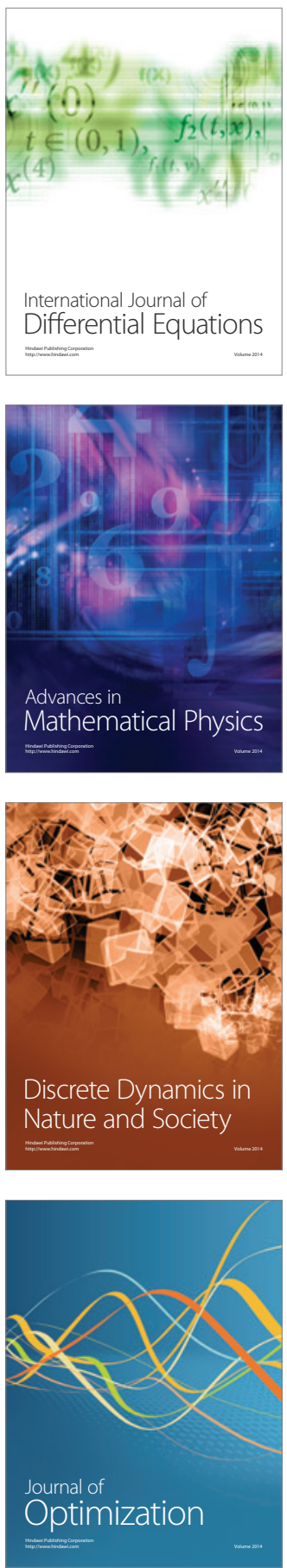